\title{
A Strictly-Positive Mass Theorem *
}

\author{
P. F. Yip \\ Department of Mathematics, University of California, San Diego, La Jolla, CA 92093, USA
}

\begin{abstract}
We show that the ADM 4-momentum of an isolated gravitational system (spatially asymptotically flat spacetime) satisfying the dominant energy condition cannot be null-like unless it is flat. Together with the positive mass theorem, this implies that the ADM 4-momentum of an isolated gravitational system must be strictly time-like.
\end{abstract}

\section{Introduction}

The total mass of an isolated gravitational system is, by now, well known to be non-negative, thanks finally to theorems of Schoen and Yau [1] and Witten [2]. More precisely, it was shown that for a spatially asymptotically flat spacetime satisfying the dominant energy condition, its ADM 4-momentum must be nonspace-like. This result was anticipated by and, in turn, confirmed the physical intuition that an isolated gravitational system is stable. However, it is interesting to observe that our physical intuition of what an isolated gravitational system should be, actually demands a stronger result, namely, the total mass must be strictly positive (equivalently, the ADM 4-momentum must be strictly time-like).

Let us first consider the situation of the Maxwell fields. One can certainly find solutions (plane waves) whose 4 momenta are null-like ${ }^{1}$. But these waves are infinite in extent and in no way can be called isolated. To form an isolated wave (i.e. a wave packet), one has to superpose plane waves with different momenta; the total 4-momentum of such a wave packet must, of course, be strictly time-like. Since general relatively in many ways resembles Maxwell theory, general features of basically the same physical concept should be common to both theories. Thus, we expect that the total 4-momentum of an isolated gravitational system must be strictly time-like. There is a caveat, though, general relativity is nonlinear. Could there exist a situation in general relativity where the nonlinearities can "glue"

* Research supported in part by NSF grant DMS84-09447

${ }^{1}$ Here, of course, the total 4-momentum is infinite. What we mean is that the field is $\alpha e^{i(E t-\mathbf{p} \cdot \mathbf{x})}$ with $E^{2}-\mathbf{p} \cdot \mathbf{p}=0$ 
together a plane wave so that its spatial extent is finite or has appropriate fall-off properties, and still making sure that the total 4-momentum is null-like. Admittedly, this kind of gluing must be extremely fine tuned and thus quite improbable. But without further investigation, we cannot dismiss, a priori, this possibility.

Finally, we would like to point out that any intuitive notion of an isolated gravitational system carries an implicit adjunct; this system has a Newtonian limit. Indeed, if an isolated system has a time-like 4-momentum, one can find classes of observers with respect to which the system is at rest (or approximately). Then standard arguments show that at large distances, these observers can well use Newtonian theory as an excellent approximation. But if an isolated system has a total 4-momentum which is null-like, there does not exist any class of observers to which the system appears approximately at rest. In fact, the system is relativistic with respect to all classes of observers. Thus, such a system does not permit a Newtonian limit. Again, while the existence of such a system does not contradict any first principles, it certainly threatens one's intuitions regarding an isolated system. The purpose of this paper is to show that the improbable is indeed impossible - a non-trivial isolated gravitational system must have a strictly timelike 4-momentum. More precisely, it will be shown that a non-flat spatially asymptotically flat spacetime satisfying the dominant energy condition must have a strictly time-like ADM 4-momentum.

We would like to mention two previous works in the literature. Witten [2], in his paper on the positive energy theorem, actually presented arguments in favor of a stronger result. Consider a spacetime satisfying the dominant energy condition with a null-like ADM momentum. It was claimed then a covariantly constant (4-dimensional) null vector $\left(k_{a}\right)$ exists (i.e. $\nabla_{a} k_{b}=0$ ). Consequently, one can employ the standard coordinates for such a spacetime (the pp waves, see Ehlers and Kundt [3]), to write down the metric which now only involves one unknown function. Finally, by imposing the appropriate fall-off properties of this metric function, Witten concluded that the spacetime must be flat. There are two points in his arguments which seem unclear. First, from the assumptions, one can easily deduce that there exists a null vector $k_{a}$ such that $D_{a} k_{b}=0$, on an asymptotically flat hypersurface $\Sigma$, where $D_{a}=h_{a}^{b} \nabla_{b}$ with $h_{a}^{b}$ the projection operator of $\Sigma$. But the procedure to lift this result off $\Sigma$ so that $\nabla_{a} k_{b}=0$ in a neighborhood of $\Sigma$ is not at all obvious. Finally, even granting that $\nabla_{a} k_{b}=0$, the coordinate system employed, a priori, bears no relation to the asymptotically flat coordinate system. Thus, one does not know how the metric component in this coordinate system should fall off. Any results based on the fall-off properties in this coordinate system are inconclusive.

Ashtekar and Horowitz [4] have also proven the theorem in question, (and also its extension to the case of null infinity) in a slightly different context. More precisely, they adopt a definition of spatially asymptotically flat spacetimes which is stronger than the usual " $3+1$ " definition (see Sect. 1). It is not clear how generic their definition is. In fact, one can find examples (though artificial) of spacetimes which are asymptotically flat in the " $3+1$ " sense but not in theirs. Moreover, the proof in [4] requires the existence of complete surfaces which have appropriate boosts properties at infinity. Since the proof of the positive energy theorem (the ADM momentum is non-space-like) can be obtained without assuming any boosts 
properties [5], it seems rather puzzling that a slightly strengthened result (that ADM momentum is strictly time-like) would require the existence of boosted surfaces. In this paper, we present a proof which (i) is valid for the more general notion of spatially asymptotically spacetimes in the " $3+1$ " sense, and (ii) is 3-dimensional and thus no "lifting" or "boosting" is required.

Synopsis of the Proof. Consider a spacetime satisfying the assumptions of the theorem (see Sect. 1 for a precise statement) with a null-like ADM 4-momentum. We (Sect. 2) can deduce the existence of a preferred null vector and thus the particular form of the 4-curvature. Then in Sect. 3, we see that this null vector induces flat 2-surfaces on which the curvature components satisfy a very simple Poisson equation. One can then easily observe (Sect. 4) that the fall-off properties of the curvature as prescribed by asymptotic flatness contradict those governed by the equation unless the spacetime is flat. The most technical part of the analysis involves relating the asymptotically flat coordinates and the natural coordinates on the flat 2-surfaces mentioned above. This part is presented in the appendix.

\section{Definitions and the Theorem}

We present here the standard definitions of asymptotic flatness. We will follow closely the definitions given by Parker and Taubes [5]. Spacetime metric $g_{a b}$ has signature $(-1,1,1,1)$.

Definition. A spacetime $\left(M, g_{a b}\right)$ is said to be spatially asymptotically flat if it admits a complete oriented 3-dimensional space-like hypersurface $\Sigma$ which is asymptotically flat i.e., there is a compact set $K \subset \Sigma$ s.t. $\Sigma-K$ is the disjoint union of a finite number of subsets $M_{i},(i=1, \ldots, k)$, the ends of $\Sigma$. Each $M_{i}$ is diffeomorphic to the compliment of a contractible compact set in $\mathbb{R}^{3}$. The induced metric on $M_{i}$ takes the form

$$
h_{i j}=\delta_{i j}+A_{i j}
$$

in the standard coordinates $\left\{x^{i}\right\}$ in $\mathbb{R}^{3}$. We impose the following fall-off conditions on both $A_{i j}$ and $K_{i j}$, the second fundamental form:

$$
\partial^{l} A_{i j}=O\left(\frac{1}{r^{l+1}}\right), \quad \partial^{l} K_{i j}=O\left(\frac{1}{r^{l+2}}\right) .
$$

Definition. $\left(M, g_{a b}\right)$ is said to satisfy the dominant energy condition if the Einstein tensor $G_{a b}$ satisfies the following property: for any future directed non-space-like vector $k_{a},-G_{a b} k^{b}$ is also a non-space-like vector.

Given a $\left(M, g_{a b}\right)$ which is asymptotically flat, one can define its ADM 4-momentum (see, for example, Arnowitt et al. [6]) for each end $M_{i}$ and $\Sigma$ as limits over spheres $S_{R, i}$ of radius $R$ in $M_{i}$ :

$$
\begin{aligned}
E_{i} & =\lim _{R \rightarrow \infty} \frac{1}{16 \pi G} \int_{S_{R, i}}\left(\partial_{j} h_{l j}-\partial_{l} h_{j j}\right) d \Omega^{l}, \\
P_{i, k} & =\lim _{R \rightarrow \infty} \frac{1}{16 \pi G} \int_{S_{R, i}} 2\left(K_{l k}-\delta_{l k} K_{j j}\right) d \Omega^{l} .
\end{aligned}
$$

We are now ready to state the main theorem of this paper. 
Theorem. ADM-4-momentum cannot be null-like. Let $\left(M, g_{a b}\right)$ be a spatially asymptotically flat spacetime satisfying the dominant energy condition, then if there exists one end $M_{i}$ s.t. $E_{i}^{2}=\sum_{k=1}^{3} P_{i, k}^{2}$, then there is only one end to $\Sigma$, and $M$ is flat along $\Sigma$.

The proof will be detailed in the following sections. Note that there is no assumption on the boost property of $\left(M, g_{a b}\right)$. Our proof is thus essentially 3-dimensional.

\section{The Form of the Curvature of $\left(M, g_{a b}\right)$ on $\Sigma$}

We want to show that for $\left(M, g_{a b}\right)$ satisfying the assumptions in the theorem, the curvature is rather restricted on $\Sigma$, namely, there exists a null vector $k_{a}$ s.t. $C_{a b c d} k^{d}$ and $R_{a b} k^{b}$ both vanish. For this result, we need to employ the spinor techniques used by Witten [2] in his proof of the positive energy theorem. We recall here some pertinent elements of this technique.

Consider a spinor $\xi^{A 2}$ on $\Sigma$ satisfying the following:

(i) $D_{A A^{\prime}} \xi^{A}=0$, where $D_{a}=h_{a}^{b} \nabla_{b}, \nabla_{b}$ is the 4-dimensional covariant derivative and $h_{a}^{b}$ is the projection operator of $\Sigma$, and

(ii) on each asymptotic and $M_{i}$ of $\Sigma, \xi^{A} \underset{r \rightarrow \infty}{\longrightarrow} \xi_{i}^{A}+O\left(\frac{1}{r}\right)^{3}$, where $\xi_{i}^{A}$ is an asymptotic constant spinor (for existence proof and a precise notion of asymptotic constant spinors, see [5] or [7]).

Taking second derivative of $D_{A A^{\prime}} \xi^{A}=0$, and integrating by parts, Witten obtained the following identity:

$$
4 \pi G \sum_{i=1}^{k} \mathbb{P}_{i}^{a} \xi_{i A} \xi_{i A}=\int_{\Sigma} d \Sigma^{a}\left\{\left(D_{b} \xi_{A}\right)\left(D^{b} \xi_{A^{\prime}}\right)+G_{a b} \xi^{B} \xi^{B^{\prime}}\right\}
$$

where $\mathbb{P}_{i}^{a}=E_{i} t^{a}+\sum_{k}^{3} P_{i, k} e_{k}^{a}, t^{a}$ is the unit normal to $\Sigma$, and $e_{k}^{a}$ is the tetrad of $h_{a b}$ on the end $M_{i}$, with $e_{k}^{a} \partial_{a} x_{l}=\delta_{k l}+O\left(\frac{1}{r}\right)$.

By using the above identity, we can easily obtain the following result.

Lemma 1. Let $\left(M, g_{a b}\right)$ be as in the theorem, then and

(i) there is only one end of $\Sigma$

(ii) there exists a spinor $\xi^{A}$ s.t. $D_{a} \xi^{A}=0$, and $\xi^{A} \underset{r \rightarrow \infty}{\longrightarrow} \xi_{0}^{A}+O\left(\frac{1}{r}\right)$ on $\Sigma$.

Proof. The proof is essentially that given by Parker and Taubes' Lemma 4.3 [5]. If for some end $M_{i}, E_{i}^{2}=\sum_{k} P_{i, k}^{2}$, then one can find a nonzero asymptotically constant

\footnotetext{
${ }^{2}$ To conform to our choice of signature for $g_{a b},(-1,1,1,1)$, we must have $g_{a b}=-\varepsilon_{A B^{\prime}} \varepsilon_{A^{\prime} B^{\prime}}$. Thus while we identify say $l^{a}$ with $\xi^{A} \xi^{A^{\prime}}$, we have $l_{a}=-\xi_{A} \xi_{A^{\prime}}$

${ }^{3}$ Convergence for spinors is defined by the norm $\left(\xi^{A}, \eta^{A}\right)=\xi^{A^{\prime}} t_{A A^{\prime}} \eta^{A}$
} 
$\xi_{i}^{A}$ on $M_{i}$ such that $\mathbb{P}_{i, a} \xi_{i}^{A} \xi_{i}^{A^{\prime}}=0$. Now let $\xi^{A}$ be the solution of $D_{A A^{\prime}} \xi^{A}=0$ on $\Sigma$ s.t. $\xi^{A} \underset{r \rightarrow \infty}{\longrightarrow} \xi_{i}^{A}+O\left(\frac{1}{r}\right)$ on $M_{i}$, but $\xi^{A} \underset{r \rightarrow \infty}{\longrightarrow} O\left(\frac{1}{r}\right)$ on all other ends. From (2.1) one immediately concludes:

$$
\int d \Sigma^{a}\left\{\left(D_{b} \xi_{A} D^{b} \xi_{A^{\prime}}\right)+G_{a b} \xi^{B} \xi^{B^{\prime}}\right\}=0 .
$$

The first term is manifestly positive definite and the second is also positive definite by the dominant energy condition. Therefore we have:

$$
D_{a} \xi_{A}=0, \text { and } G_{a b} \xi^{B} \xi^{B^{\prime}}=0 .
$$

Thus (ii) is established. To obtain (i), we simply note that $D_{a} \xi_{A}=0$ is incompatible with the asymptotic conditions namely $\xi^{A} \sim \xi_{i}^{A}+O\left(\frac{1}{r}\right)$ on $M_{i}$ but $\xi^{A} \sim O\left(\frac{1}{r}\right)$ on all other ends, unless there is only one end $M_{i}$.

We can now proceed to deduce the form of the curvature of $\left(M, g_{a b}\right)$ on $\Sigma$.

Lemma 2. Let $\left(M, G_{a b}\right)$ be as in the theorem, then on $\Sigma$, we have the following form for the Weyl and Ricci curvature, and

(i) $R_{a b}=\alpha l_{a} l_{b}, \alpha \geqq 0$, (where $l_{a}=-\xi_{A} \xi_{A^{\prime}}, \xi_{A}$ is the spinor obtained in Lemma 1),

(ii) $C_{a b c d}=f \xi_{A} \xi_{B} \xi_{C} \xi_{D^{\prime}} \varepsilon_{A^{\prime} B^{\prime}} \varepsilon_{C^{\prime} D^{\prime}}+$ c.c., for some $f$.

Proof. (i) is relatively trivial. From the proof of Lemma 1, we have $G_{a b} l^{b}=0$. For any nonspacelike $P^{a}$, we then have $P^{a} G_{a b} l^{b}=0$. But since $P^{a} G_{a b}$ is non-space-like by the dominant energy condition, we must have $P^{a} G_{a[b} l_{c]}=0$. But since $P^{a}$ is an arbitrary non-space-like vector, we conclude $G_{a[b} l_{c]}=0$, and by symmetry $G_{a b}=\alpha l_{a} l_{b}$ for some $\alpha$. Then we have $R_{a b}=\alpha l_{a} l_{b}$. Finally $\alpha \geqq 0$ by the dominant energy condition.

One can prove (ii) by straightforward computation. Expanding $D_{[a} D_{b]} \xi_{c}=0$, in terms of spinors one has [with (i)],

$$
\left(\delta_{a}^{m}+t_{a} t^{m}\right)\left(\delta_{b}^{n}+t_{b} t^{n}\right)\left(\varepsilon_{M^{\prime} N^{\prime}} \Psi_{M N C D} \xi^{D}\right)=0 .
$$

One further obtains,

$$
\varepsilon_{A^{\prime} B^{\prime}} \Psi_{A B C D} \xi^{D}+t_{b} t^{m} \varepsilon_{A^{\prime} M^{\prime}} \Psi_{A M C D} \xi^{D}+t_{a} t^{m} \varepsilon_{M^{\prime} B^{\prime}} \Psi_{M B C D} \xi^{D}=0
$$

Finally, contracting with $\varepsilon^{A^{\prime} B^{\prime}}$ gives

$$
\Psi_{A B C D} \xi^{D}=0
$$

But this implies (ii).

\section{3. " $2+1$ " Foliation of $\Sigma$ at $\infty$}

We will now show that the spinor $\xi^{A}$ obtained from last section actually induces two surfaces which foliate $\Sigma-K$, where $K$ is compact. These two surfaces turn out 
to be flat planes. Interestingly, by Bianchi identities, the functions $\alpha$ and $f$ occurring in the curvature satisfy a Poisson equation on each plane. This equation relating $\alpha$ and $f$ will be crucial in determining their asymptotic behaviors (Sect. 4).

To facilitate our discussion, we will simplify the asymptotic conditions. Without loss of generality, the asymptotically flat coordinates $(x, y, z)$ are chosen to have the following properties. Consider the asymptotically constant spinor $\xi_{0}^{A}$ (in Lemma 1), we can find $\eta^{A}$, another asymptotically constant spinor such that $\eta^{A} \xi_{A}=1$. Now, let $x^{a}, y^{a}, z^{a}, t^{a}$ be the tetrad associated with the asymptotically flat coordinates $x, y, z$ on $\Sigma\left[\right.$ i.e., $x^{a} \partial_{a} x=1+O\left(\frac{1}{r}\right), x^{a} \partial_{a} y=O\left(\frac{1}{r}\right)$, etc. $]$. Then we require that $\quad \xi_{0}^{A} \xi^{A^{\prime}}=\frac{1}{\sqrt{2}}\left(t^{a}+z^{a}\right), \quad \underset{0}{\eta^{A} \eta^{A^{\prime}}}=\frac{1}{\sqrt{2}}\left(t^{a}-z^{a}\right) \quad$ and $\xi_{0}^{A} \eta^{A^{\prime}}=\frac{1}{\sqrt{2}}\left(x^{a}+i y^{a}\right)=\underset{0}{m^{a}}$

We begin with the following simple result.

Lemma 3. Let $l^{a}=\xi^{A} \xi^{A^{\prime}}$, with $\xi^{A}$ given in Lemma 1, and let $S_{a}=h_{a}^{b} l_{b}$, $h_{a}^{b}$ being the projection operator of $\Sigma$. Then $\mathscr{D}_{[a} S_{b]}=0$ on $\Sigma$, where $\mathscr{D}_{a}$ is the covariant derivative of $h_{a b}$.

Proof. By straightforward computation, $D_{a} S_{b}=D_{a} h_{b}^{c} l_{c}$. But since $D_{a} l_{c}=0$ by Lemma 1, we have $D_{a} S_{b}=\left(D_{a} h_{b}^{c}\right) l_{c}$ or $h_{b}^{c} D_{a} S_{c}=K_{a b}\left(t^{c} l_{c}\right)$. But the left-hand side is precisely $\mathscr{D}_{a} S_{b}$, and $K_{a b}$ is symmetric. Thus $\mathscr{D}_{[a} S_{b]}=0$.

The 2-surfaces we are searching for are those orthogonal to $S_{a}$. But to simplify arguments concerning asymptotics, it would be helpful to have a global control over these surfaces, such as they are level surfaces of some function. This would only be true if $\Sigma$ is simply connected. In general, however, we do have such a control for the region of $\Sigma$ that concerns us, the asymptotic region. Let $B_{R}$ be the region of $\Sigma$ inside the sphere of radius $R$ (in asymptotically flat coordinates). Then $A=\Sigma-B_{R}$ is simply connected and thus $S_{a}=\partial_{a} S$ for some $S$ in $A$. Next we would like to determine the geometry and topology of the level surfaces given by $S$. For this and later purposes, we need the following construction.

Constructions. Let $\xi^{A}$ be given in Lemma 1. Then let $\eta^{A}$ be such that: and

(i) $\eta^{A} \xi_{A}=1$

(ii) $\eta^{A} \eta^{A^{\prime}}, \xi^{A} \xi^{A^{\prime}}$ and $t^{a}$ of $\Sigma$ are coplanar or equivalently, $\eta^{A} \xi^{A^{\prime}} t_{A A^{\prime}}=0$. We see $\eta^{A}$ is determined uniquely by (i) and (ii).

The asymptotic behavior of $\eta^{A}$ follows from that of $\xi^{A}$. Since $\xi^{A} \sim \xi_{0}^{A}+O\left(\frac{1}{r}\right)$, one can easily see that $\eta^{A} \sim \underset{0}{\eta^{A}}+O\left(\frac{1}{r}\right)$. Now let $m^{a}=\xi^{A} \eta^{A^{\prime}}$. One further sees that the following asymptotic conditions hold: $S^{a} \sim \frac{z^{a}}{\sqrt{2}}+O\left(\frac{1}{r}\right)$ and $m^{a} \sim \frac{1}{\sqrt{2}}\left(x^{a}+i y^{a}\right)$ $+O\left(\frac{1}{r}\right)$. 
Lemma 4. Let $S_{a}$ be given in Lemma 3, and $m^{a}$ as defined above, then:

(i) $m^{a} S_{a}=0, m^{a} t_{a}=0, m^{a} m_{a}=0$, and $m^{a} \bar{m}_{a}=1$

and

(ii) $\left(\mathscr{D}_{a} m_{[b}\right) S_{c]}=0$, when $\mathscr{D}_{a}$ is the covariant derivative of $h_{a b}$.

Proof. (i) follows trivially from the definitions. To prove (ii), consider $D_{a} \eta^{B}$. Since $D_{a}\left(\eta^{B} \xi_{B}\right)=0$, and $D_{a} \xi_{B}=0$, we must have $\left(D_{a} \eta_{[B}\right) \xi_{C]}=0$. Thus $\left(D_{a} m_{[b}\right) l_{c]}=0$. On projecting with $h_{a b}$, we immediately have (ii).

Since $S_{a}$ is hypersurface orthogonal by Lemma 3, we have the following result.

Lemma 5. Let $\mathscr{K}$ be a 2-surface orthogonal to $S_{a}$, with the induced metric $j_{a b}=h_{a b}-\beta S_{a} S_{b}$, where $\beta=\left(S_{a} S^{a}\right)^{-1} \cdot \mathscr{K}$ is flat.

Proof. Let $\Delta_{a}$ be the covariant derivative associated with $j_{a b}$. Then all we need to do is to find two independent vector fields on $\mathscr{K}$ which are covariantly constant with respect to $\Delta_{a}$. Now, $m^{a}$ and $\bar{m}^{a}$ (complex conjugate of $m^{a}$ ) indeed satisfy $\Delta_{a} m^{b}=\Delta_{a} \bar{m}^{b}=0$. Consider $\Delta_{a} m^{b}=j_{e}^{b} j_{a}^{c} \mathscr{D}_{c} m^{e}$. But by Lemma 4, $\left(\mathscr{D}_{c} m^{[e}\right) S^{d]}=0$, therefore $j_{e}^{b} \mathscr{D}_{c} m^{e}=0$. Thus $\Delta_{a} m^{b}=0$. By Lemma $4, m^{a}$ and $\bar{m}^{a}$ both are vector fields on $\mathscr{K}$. The two real vector fields, $\left(m^{a}+\bar{m}^{a}\right)$ and $i\left(m^{a}-\bar{m}^{a}\right)$ can easily be seen to be independent, and from above, are covariantly constant.

In particular the level surfaces of $S$ in $A$ are all flat with the induced metric.

Next we would want to determine the topology of $S$. We will show that $S=$ const surfaces are either flat 2-planes or portions of flat 2-planes. To do that, we just need to show that any level surface of $S$, upon maximally extended, is simply connected. Let $S^{\prime}$ be the maximally extended 2 surfaces of $S$ which are orthogonal to $S_{a}$. One can achieve the maximal extension to an $S=$ const surface by the following. Take the union of all connected without boundary 2-surfaces orthogonal to $S_{a}$ and containing $S=$ const as a subset. It is easy to see that this union is also a 2-surface orthogonal to $S_{a}$ and containing $S=$ const. The existence of $S^{\prime}$ does not imply that $\Sigma$ as a whole is foliated, there might be points inside $B_{R}$ which are not on any $S^{\prime}$. But in $\left(\Sigma-B_{R}\right)$, every point is on a $S=$ const (thus $S^{\prime}$ ) surface.

Lemma 6. $S^{\prime}$ are flat 2-planes.

Proof. All we need to show is $S^{\prime}$ are simply connected. Let us assume the contrary and arrive at a contradiction. Take a single $S^{\prime}$ which is not simply connected. First we note that $S^{\prime}$ can be shown to be complete. The set of non-contractible curves associated with a point $p \in S^{\prime}$ must be bounded below in length. Take a sequence of minimizing curves out of this set. We can obtain a limit curve $C$ attaining the greatest lower bound in length. It is obvious that $C$ is also incontractible. But since any variation of $C$ would produce a longer curve, $C$ must be a geodesic. Thus we can find a constant $\beta$ such that $\beta m^{a}+\bar{\beta} \bar{m}^{a}=n^{a}$ generates $C$. Now we can parallel transport $C$ out to large $r$ ( $r$ is unbounded on $S^{\prime}$, see appendix). But we know at large $r$ we have $m^{a} \sim \underset{0}{m^{a}}+O\left(\frac{1}{r}\right)$. Since $\bar{m}_{0}^{a}\left(\partial_{a} x+i \partial_{a} y\right) \sim \sqrt{2}+O\left(\frac{1}{r}\right)$. Therefore $n^{a}\left(\beta\left(\partial_{a} x+i \partial_{a} y\right)+\right.$ c.c. $)=2 \sqrt{2} \beta \bar{\beta}+O\left(\frac{1}{r}\right)$. But since $\beta(x+i y)+$ c.c. is a continuous function, we must have $n^{a} \partial_{a}(\beta(x+i y)+$ c.c. $)=0$ somewhere on $C$, a contradiction. 
To finish this section, we want to obtain a differential equation relating $f$ and $\alpha$ in the 4-curvature valid on any $S^{\prime}$. To this end, we first rewrite the curvature. On $\Sigma$, we have by Lemma 1.

$$
\begin{aligned}
C_{a b c d} & =f \xi_{A} \xi_{B} \xi_{C} \xi_{D^{\prime}} \varepsilon_{A^{\prime} B^{\prime}} \varepsilon_{C^{\prime} D^{\prime}}+\text { c.c. }, \\
R_{a b} & =\alpha l_{a} l_{b}, \text { where } l_{a}=-\xi_{A} \xi_{A^{\prime}} .
\end{aligned}
$$

Now, we can use $m^{a}$ to write:

$$
C_{a b c d}=4 f m_{[a} l_{b]} m_{[c} l_{d]}+\text { c.c. . }
$$

Thus, on $\Sigma$, we have the following form for the Riemann tensor.

$$
R_{a b c d}=4 f m_{[a} l_{b]} m_{[c} l_{d]}+\text { c.c. }+\alpha\left(g_{a[c} l_{d]} l_{b}-g_{b[c} l_{d]} l_{a}\right) .
$$

Applying $D_{e}$, we have:

$$
D_{e} R_{a b c d}=4 D_{e} f\left(m_{[a} l_{b]} m_{[c} l_{d]}\right)+\text { c.c. }+D_{e} \alpha\left(g_{a[c} l_{d]} l_{b}-g_{b[c} l_{d]} l_{a}\right) .
$$

The above equation is valid because $D_{e}=h_{e}^{a} \nabla_{a}$ is a derivative along $\Sigma$, on which the special form of the curvature holds. On the other hand, replacing $D_{e}$ by $\nabla_{e}$ would not be legal in general. Contract with $\bar{m}^{e}$ and $m^{a}$, we obtain:

$$
m^{a} \bar{m}^{e} D_{e} R_{a b c d}=2 l_{b} \bar{m}_{[c} l_{d]} \bar{m}^{e} D_{e} \bar{f}+\bar{m}^{e} D_{e} \alpha\left(m_{[c} l_{d]} l_{b}\right) .
$$

Next, we consider

$$
h_{f}^{b} m^{a} \bar{m}^{e}\left(D_{e} R_{a b c d}+D_{a} R_{b e c d}+D_{b} R_{e a c d}\right) .
$$

Since both $m^{a}$ and $\bar{m}^{e}$ are in $\Sigma$, we can replace $D_{a}$ in the above expression by $\nabla_{a}$. But, by Bianchi identity, the above expression vanishes identically. On simplifying, one finally obtains the following equation

$$
2 \bar{m}^{e} D_{e} \bar{f}-m^{a} D_{a} \alpha=0 .
$$

\section{Fall-Off Properties of the 4-Curvature}

We now simply use $(*)$ to deduce the asymptotic behavior of $f$ and $\alpha$. The equation $(*)$ is a Poisson equation in 2-dimensions, with $f$ as the potential and $\alpha$ supplying a quadrupole source. The key to the analysis is that $\alpha$ is non-negative, and thus quadrupole moment is non-negative. Therefore, $f$ should fall of as $\frac{1}{r^{2}}$ on the 2-surface $S^{\prime}$. But this will contradict that prescribed by asymptotic flatness, thus $f$ and $\alpha$ both have to vanish on $S^{\prime}$.

Let us begin by obtaining the desired Poisson equation on $S^{\prime}$. First, note that we can replace $D_{e}$ by $\Delta_{e}$ in $(*)$ and obtain an equation on $S^{\prime}$.

$$
2 \bar{m}^{e} \Delta_{e} \bar{f}-m^{e} \Delta_{e} \alpha=0 .
$$

Operating by $m^{a} \Delta_{a}$, we have

$$
2 m^{a} \Delta_{a} \bar{m}^{e} \Delta_{e} \bar{f}-m^{a} \Delta_{a} m^{e} \Delta_{e} \alpha=0 .
$$


Since $S^{\prime}$ is a complete flat 2 surface, we can define coordinates $\tilde{x}$ and $\tilde{y}$ on $S^{\prime}$ s.t. $m_{a}$ $=\frac{1}{\sqrt{2}}\left(\partial_{a} \tilde{x}+i \partial_{a} \tilde{y}\right)$ etc. The above equation becomes

$$
2\left(\frac{\partial^{2}}{\partial \tilde{x}^{2}}+\frac{\partial^{2}}{\partial \tilde{y}^{2}}\right) R=\left(\frac{\partial^{2}}{\partial \tilde{x}^{2}}-\frac{\partial^{2}}{\partial \tilde{y}^{2}}\right) \alpha
$$

and

$$
\left(\frac{\partial^{2}}{\partial \tilde{x}^{2}}+\frac{\partial^{2}}{\partial \tilde{y}^{2}}\right) I=-\frac{\partial^{2}}{\partial \tilde{x} \partial \tilde{y}} \alpha
$$

where $R$ and $I$ are the real and imaginary part of $f$ respectively.

First of all, we will show that in general, $\alpha$ must vanish on $S^{\prime}$.

Lemma 7. Given the conditions in the theorem, $\alpha=0$ on any $S^{\prime}$.

Proof. The key is to recognize that if $\alpha \neq 0$ on $S^{\prime}, \int_{S^{\prime}} \alpha>0$ by dominant energy condition. This means that there is a positive quadrupole moment. The potential ( $R$ and $I$ ) would fall off as $\frac{1}{\tilde{r}^{2}}$. But the asymptotic conditions require that $R$ and $I$ fall off like $\frac{1}{\tilde{r}^{3}}$. Thus we have a contradiction unless $\alpha=0$. More precisely, consider

$$
2 \int_{S^{\prime}}\left(\tilde{x}^{2}-\tilde{y}^{2}\right)\left(\frac{\partial}{\partial \tilde{x}^{2}}+\frac{\partial}{\partial \tilde{y}^{2}}\right) R=\int_{S^{\prime}}\left(\tilde{x}^{2}-\tilde{y}^{2}\right)\left(\frac{\partial^{2}}{\partial \tilde{x}^{2}}-\frac{\partial^{2}}{\partial \tilde{y}^{2}}\right) \alpha .
$$

The integrals exist by the asymptotic fall-off conditions (see Proposition 3, Appendix). Integrating by parts, the left-hand side vanishes identically, while the right-hand side becomes $2 \int_{S^{\prime}} \alpha d \tilde{x} d \tilde{y}$. Thus $\int_{S^{\prime}} \alpha d \tilde{x} d \tilde{y}=0$. But since $\alpha$ is non-negative by the dominant energy condition, $\alpha=0$.

Lemma 8. Given the conditions in the theorem, if $\alpha=0$ on $S^{\prime}\left(R_{a b}=0\right)$, then $f=0\left(R_{a b c d}=0\right)$ on $S^{\prime}$.

Proof. The first key is to recognize the asymptotic behavior of $f$. This is done in Proposition 3 of the Appendix. We will include a different argument here for completeness. First, we have

$$
C_{a b c d}=4 f m_{[a} l_{b]} m_{[a} l_{b]}+\text { c.c. . }
$$

Now since $m^{a} \sim \frac{1}{\sqrt{2}}\left(x^{a}+i y^{a}\right)+O\left(\frac{1}{r}\right)$ and $l^{a} \sim \frac{1}{\sqrt{2}}\left(t^{a}+z^{a}\right)+O\left(\frac{1}{r}\right)$, we have

$$
\begin{aligned}
C_{x z x z} & =\frac{1}{2}(f+\bar{f})\left(1+O\left(\frac{1}{r}\right)\right), \\
C_{y z y z} & =\frac{1}{2} i(f-\bar{f})\left(1+O\left(\frac{1}{r}\right)\right), \\
f & =\left(C_{x z x z}-i C_{y z y z}\right)\left(1+O\left(\frac{1}{r}\right)\right) .
\end{aligned}
$$


Now the curvature components in asymptotic flat coordinates by the conditions on the fall off of $h_{i j}$ and $K_{i j}$, must fall off like $O\left(\frac{1}{r^{3}}\right)$. Therefore $f \sim O\left(\frac{1}{r^{3}}\right)$.

The second key step is to recognize that on $S^{\prime}$, along any radial direction, $B<\frac{\tilde{r}}{r}<A$ for some $A, B>0$ (see Proposition 2, Appendix). Thus $f \sim O\left(\frac{1}{\tilde{r}^{3}}\right)$. Now since $\alpha=0$, we have simply, $\left(\frac{\partial^{2}}{\partial \tilde{x}^{2}}+\frac{\partial^{2}}{\partial \tilde{y}^{2}}\right) f=0$, or $f$ is harmonic on $S^{\prime}$. Together with the condition that $f \sim O\left(\frac{1}{\tilde{r}^{3}}\right)$ we must have $f=0$ on $S^{\prime}$ identically.

Now, we can complete the proof of the theorem. Take any point $x \in \Sigma-B_{R}$, for some large $R$. Then we can find $S^{\prime}$ s.t. $x \in S^{\prime}$. But on $S^{\prime}, \alpha=0$ and $f=0$, or $R_{a b c}^{d}=0$. Thus $R_{a b c}^{d}=0$ in $\Sigma-B_{R}$. This implies that $\mathbb{P}_{A D M}^{a}=0$. Now, we appeal to the positive energy theorem $[1,2,5]$, to conclude that $R_{a b c}^{d}=0$ on $\Sigma$, thus establishing our theorem.

\section{Conclusion and Discussion}

Thus we have strengthened the positive mass theorem to conclude that a nontrivial isolated gravitational system must have a strictly time-like 4-momentum. The central feature of our proof is that it is essentially 3-dimensional. At the same time, the fact that $\Sigma$ is embedded in a 4-dimensional space is also crucial. For example, we take the 4-dimensional Bianchi identities, and study them on $\Sigma$ via projection. This procedure is perhaps not surprising in retrospect. Since our statement calls for a relation between energy and momentum, which are dictated by the intrinsic geometry and the imbedding of $\Sigma$ respectively, it only seems natural to study equations which do reflect both features of $\Sigma$.

\section{Appendix}

Here, we present the technical results which establish the asymptotic fall-off behavior of the 4-curvature. Again, let $S^{\prime}$ be as defined in Sect. 3.

Proposition 1. The function $r=\left(x^{2}+y^{2}+z^{2}\right)^{1 / 2}$, with $x, y, z$ the asymptotic coordinates of $\Sigma$, is unbounded on $S^{\prime}$.

Proof. Since $S \subset S^{\prime}$, we only need to show that $S=$ const $=C$, say, has the stated property. Assume the contrary, i.e. $\exists r_{0}$ s.t. $r<r_{0}$ on $S$. Take $r_{0}$ large enough so that the asymptotic conditions $\left(\frac{\partial}{\partial z}\right)^{a} S_{a}=\frac{1}{\sqrt{2}}+O\left(\frac{1}{r}\right) \geqq \varepsilon>0$ holds for $r>r_{0}$. Now consider the 3-plane $L:\left(x=r_{0}\right)$. On $L$, we have $r \geqq r_{0}$, therefore the estimate $\left(\frac{\partial}{\partial z}\right)^{a} S_{a} \geqq \varepsilon>0$ holds on $L$. Then $\frac{\partial S}{\partial z} \geqq \varepsilon>0$ on $L$. Thus along any integral curve of $\left(\frac{\partial}{\partial z}\right)^{a}$ on $L, S \geqq \varepsilon z+S_{0}$ for $z \geqq 0$ and $S \leqq \varepsilon z+S_{0}$ for $z \leqq 0$, for some $S_{0}$. Therefore $S$ takes on the value $C$ for some $z$ on $L$. But $r \geqq r_{0}$ on $L$, a contradiction. 
We now establish a result relating the natural coordinates on $S^{\prime}$ (Sect. 4) and $r$. Proposition 2. On $S^{\prime}, \exists$ constants $A, B$, and $\tilde{R}_{0}$, such that for $\tilde{r}>\widetilde{R}_{0}, B \leqq \frac{\tilde{r}}{r} \leqq A$.

Proof. There are two steps to the proof. The first is to show that along any radial direction of $\tilde{r}, r$ becomes unbounded. The second step is then to use the asymptotic estimates for $\tilde{x}$ and $\tilde{y}$ in terms of $x$ and $y$, which are valid for large $r$, to deduce the precise relation between $\tilde{r}$ and $r$.

For the first step, it turns out to be convenient to consider the tube $T$. $\left\{x^{2}+y^{2}=C^{2}\right\}$. We want to show that for some $C, T$ intersects $S^{\prime}$ in a closed curve, homeomorphic to $S^{1}$. Let us take $C$ large enough s.t. for $r \geqq C$, the following estimates hold: $\quad \tilde{x}^{a} \partial_{a} x=1+O\left(\frac{1}{r}\right), \quad \tilde{y}^{a} \partial_{a} y=1+O\left(\frac{1}{r}\right), \quad \tilde{x}^{a} \partial_{a} y=O\left(\frac{1}{r}\right)$, $\tilde{y}^{a} \partial_{a} x=O\left(\frac{1}{r}\right)$, and $\left(\frac{\partial}{\partial z}\right)^{a} S_{a}=\frac{1}{\sqrt{2}}+O\left(\frac{1}{r}\right)$. Repeating essentially the same argument as in Proposition 1, we have for any $x, y \in T, \exists$ unique $z(x, y)$ s.t. the point $(x, y, z(x, y)) \in S^{\prime}$. It's also easy to see that $z(x, y)$ is continuous. Thus $K=T \cap S^{\prime}$ $=(x, y, z(x, y))$, with $x^{2}+y^{2}=C^{2}$, is a curve homeomorphic to $S^{1}$. Without loss of generality, we take the origin of the $\tilde{x}$ and $\tilde{y}$ coordinate system of $S^{\prime}$ to be in the region bounded by $K(\operatorname{int} K)$. Now, we want to show that in the region exterior to $K, x^{2}+y^{2}>C^{2}$. This can be seen as follows. $T$ divides $\Sigma$ into two disconnected open sets, namely one with $x^{2}+y^{2}>C^{2}$, and its complement. Likewise, $T$ divides $S^{\prime}$ into two disconnected components (namely the interior and exterior of $K$ ). Now since $x^{2}+y^{2}$ is unbounded on $S^{\prime}$, and since the interior of $K$ has compact closure, the exterior of $K$ must have non-empty intersection with the region of $\Sigma$ s.t. $x^{2}+y^{2}>C^{2}$. Since exterior of $K$ is connected, it cannot intersect the region of $\Sigma$ with $x^{2}+y^{2}<C^{2}$. Thus $x^{2}+y^{2}>C^{2}$ in the exterior of $K$. In particular, let $\tilde{r}_{\max }=\max \tilde{r}$ on int $K$. Then for $\tilde{r} \geqq \tilde{r}_{\max } \cdot r \geqq\left(x^{2}+y^{2}\right)^{1 / 2} \geqq C$.

We now proceed with the second step. Consider the case where $\tilde{x}=\tilde{r}$. Then in the region $\tilde{r} \geqq \tilde{r}_{\max }$, we have $\tilde{x}^{a} \partial_{a} x=1+O\left(\frac{1}{r}\right), \tilde{x}^{a} \partial_{a} y=O\left(\frac{1}{r}\right)$, and $\tilde{x}^{a} \partial_{a} z=O\left(\frac{1}{r}\right)$. (The estimates are valid for $r \geqq C$ in this region.) Thus one can find $1>\varepsilon>0$ s.t.

$$
1-\varepsilon \leqq \tilde{x}^{a} \partial_{a} x \leqq 1+\varepsilon, \quad-\varepsilon \leqq \tilde{x}^{a} \partial_{a} y \leqq \varepsilon, \quad-\varepsilon \leqq \tilde{x} \partial_{a} z \leqq \varepsilon .
$$

Then along the ray $\tilde{x}=\tilde{r}$, we have

$$
\begin{gathered}
(1-\varepsilon)\left(\tilde{x}-\tilde{x}_{0}\right) \leqq x-x_{0} \leqq(1+\varepsilon)\left(\tilde{x}-\tilde{x}_{0}\right), \\
-\varepsilon\left(\tilde{x}-\tilde{x}_{0}\right) \leqq y-y_{0} \leqq \varepsilon\left(\tilde{x}-\tilde{x}_{0}\right), \\
-\varepsilon\left(\tilde{x}-\tilde{x}_{0}\right) \leqq z-z_{0} \leqq \varepsilon\left(\tilde{x}-\tilde{x}_{0}\right) .
\end{gathered}
$$

Squaring and summing, we obtain

$$
(1-\varepsilon)^{2}\left(\tilde{x}-\tilde{x}_{0}\right)^{2} \leqq\left(x-x_{0}\right)^{2}+\left(y-y_{0}\right)^{2}+\left(z-z_{0}\right)^{2} \leqq\left((1+\varepsilon)^{2}+2 \varepsilon^{2}\right)\left(\tilde{x}-\tilde{x}_{0}\right)^{2} .
$$

Generalize to any radial directions, we obtain

$$
(1-\varepsilon)^{2}\left(\tilde{r}-\tilde{r}_{0}\right)^{2} \leqq\left|\mathbf{r}-\mathbf{r}_{0}\right|^{2} \leqq\left((1+\varepsilon)^{2}+2 \varepsilon^{2}\right)\left(\tilde{r}-\tilde{r}_{0}\right)^{2} .
$$


The following two inequalities are easily obtained:

$$
(1-\varepsilon)\left(\tilde{r}-\tilde{r}_{0}\right) \leqq|| \mathbf{r}|+| \mathbf{r}_{0}||, \quad|\mathbf{r}|-\left|\mathbf{r}_{0}\right| \leqq\left((1+\varepsilon)^{2}+2 \varepsilon^{2}\right)\left(\tilde{r}-\tilde{r}_{0}\right) .
$$

Let $r_{\max }=\max \left|\mathbf{r}_{0}\right|$ on $\tilde{r}=\tilde{r}_{\max }$. Then we have

$$
\begin{aligned}
(1-\varepsilon)\left(\tilde{r}-\tilde{r}_{0}\right) & \leqq r+r_{\max } \\
r-r_{\max } & \leqq\left((1+\varepsilon)^{2}+2 \varepsilon^{2}\right)\left(\tilde{r}-\tilde{r}_{0}\right) .
\end{aligned}
$$

It is now obvious that for sufficiently large $\widetilde{R}_{0}$, we can find two constants $A$ and $B>0$ s.t. for $\tilde{r} \geqq \widetilde{R}_{0}, A \geqq \frac{\tilde{r}}{r} \geqq B$.

Proposition 3. On $S^{\prime}$, the following asymptotic conditions hold for $f$ and $\alpha$.

(i) $f, \alpha=O\left(\frac{1}{\tilde{r}^{3}}\right)$

(ii) $\frac{\partial}{\partial \tilde{x}}, \frac{\partial}{\partial \tilde{y}}$ of $f$ or $\alpha=O\left(\frac{1}{\tilde{r}^{4}}\right)$

and

(iii) $\frac{\partial^{2}}{\partial \tilde{x}^{2}}, \frac{\partial^{2}}{\partial \tilde{y}^{2}}, \frac{\partial^{2}}{\partial \tilde{x} \partial \tilde{y}}$ of $f$ or $\alpha=O\left(\frac{1}{\tilde{r}^{5}}\right)$.

Proof. We know, by definitions, that the 4-curvature components in asymptotic coordinates $(x, y, z)$ have the desired fall-offs on $\Sigma$. We need only to translate those conditions to $f$ and $\alpha$, in terms of $\tilde{x}$ and $\tilde{y}$ on $S^{\prime}$. We will present arguments for $\alpha$, the case for $f$ is similar. First, we show that $\alpha$ has the right fall-off in the $(x, y, z)$ system. By the initial value constraint equation, one has

$$
G_{a b} t^{a} t^{b}=\frac{1}{2}\left\{{ }^{(3)} R+\left(K_{a}^{a}\right)^{2}-K_{a b} K^{a b}\right\} .
$$

The left-hand side is simply $\alpha\left(l_{a} t^{a}\right)^{2}=\alpha \beta^{2}$. Since $l_{a}$ goes as $l_{0}+O\left(\frac{1}{r}\right)$, we have $\beta^{2}=1$ $+O\left(\frac{1}{r}\right)$. The right-hand side by asymptotic conditions goes as $O\left(\frac{1}{r^{3}}\right)$, we easily have $\alpha=O\left(\frac{1}{r^{3}}\right)$. To obtain fall-off for $\frac{\partial \alpha}{\partial x^{i}}$ and $\frac{\partial^{2} \alpha}{\partial x^{i} \partial x^{j}}\left(x^{1}=x, x^{2}=y, x^{3}=z\right)$, we note that if $\frac{\partial \beta}{\partial x^{i}}=O\left(\frac{1}{r^{2}}\right)$ and $\frac{\partial^{2} \beta}{\partial x^{i} \partial x^{j}}=O\left(\frac{1}{r^{3}}\right)$, we will have the desired fall off for $\alpha$, namely $\frac{\partial \alpha}{\partial x^{i}}=O\left(\frac{1}{r^{4}}\right)$ and $\frac{\partial^{2} \alpha}{\partial x^{i} \partial x^{j}}=O\left(\frac{1}{r^{5}}\right)$. Now $\beta=l^{a} t_{a} \cdot D_{a} \beta=l^{b} K_{a b}$. Thus $\left|D_{a} \beta\right| \leqq\left|l^{b}\right|\left|K_{a b}\right| \leqq \beta O\left(\frac{1}{r^{2}}\right) \leqq O\left(\frac{1}{r^{2}}\right)$. Thus $\frac{\partial \beta}{\partial x^{i}}=O\left(\frac{1}{r^{2}}\right)$. Similarly, we get $\frac{\partial^{2} \beta}{\partial x^{i} \partial x^{j}}$ $=O\left(\frac{1}{r^{3}}\right)$. Finally, we want to obtain the fall off in terms of $\tilde{x}$ and $\tilde{y}$. Consider on $S^{\prime}$, $\left|m^{a} D_{a} \alpha\right| \leqq\left|m^{a}\right|\left|D_{a} \alpha\right|$. But $\left|m^{a}\right|=1, \quad \therefore\left|m^{a} D_{a} \alpha\right|=O\left(\frac{1}{r^{4}}\right)$. Similarly, $\left|m^{a} D_{a} m^{b} D_{b} \alpha\right|$ $=O\left(\frac{1}{r^{5}}\right)$. Now we only need to appeal to Proposition 2 to arrive at our conclusion. 
Acknowledgements. I would like to thank A. Ashtekar and D. Christodoulou for many helpful discussions. My thanks also go to D. Brill, A. Sen, R. Sorkin, S.-T. Yau, and the referee for suggesting the simple proof of Lemma 2.

\section{References}

1. Schoen, R., Yau, S.-T.: The proof of the positive mass theorem. II. Commun. Math. Phys. 9, 231-260 (1981)

2. Witten, E.: A new proof of the positive energy theorem. Commun. Math. Phys. 80, 381-402 (1981)

3. Ehlers, J., Kundt, W.: Exact solutions of the gravitational field equations. In: Gravitation: an introduction to current research. Witten, L. (ed.). New York: Wiley 1962

4. Ashtekar, A., Horowitz, G.: Energy-momentum of isolated systems cannot be null. Phys. Lett. 89 A, 181-184 (1982)

5. Parker, T., Taubes, C.H.: On Witten's proof of the positive energy theorem. Commun. Math. Phys. 84, 223-238 (1982)

6. Arnowitt, R., Deser, S., Misner, C.W.: The dynamics of general relativity. In: Gravitation: an introduction to current research. Witten, L. (ed.). New York: Wiley 1962

7. Reula, O.: Existence theorem for solutions of Witten's equation and nonnegativity of total mass. J. Math. Phys. 23, 810-814 (1982)

Communicated by S.-T. Yau

Received June 12, 1986; in revised form September 19, 1986 
